\title{
The chemotactic response of blood neutrophils and monocytes to strains of Escherichia coli with different virulence characteristics
}

\author{
W. BEEKEN, J. FABIAN and J. FENWICK*
}

Departments of Medicine and Biometry, University of Vermont, Burlington, Vermont, 05405-0068, USA

\begin{abstract}
Summary. Chemotactic responses of blood neutrophils and monocytes to media conditioned by eight strains of Escherichia coli with different virulence characteristics were measured in modified Boyden assay chambers to determine if these characteristics were associated with differences in leucocyte mobility. Responding neutrophils and monocytes were prepared on conventional density gradients, and in three instances, the chemotaxis of eosinophils isolated on metrizamide gradients was also studied. Media conditioned by enteroinvasive and nonenteroinvasive $E$. coli strains were tested as chemo-attractants and compared to the formylated peptide standard attractant. Chemotactic activity of neutrophils was greater than that of monocytes and eosinophils, and migration by all populations was significantly greater to conditioned media than to the control medium. Chemotactic responses to media conditioned by non-enteroinvasive $E$. coli and strains lacking virulence factors was greater than to media conditioned by plasmid- and Sereny-positive enteroinvasive organisms. The results suggest that virulence factors of $E$. coli that determine invasiveness did not augment the chemotactic responses of the leucocyte populations tested in vitro, and give no support to the hypothesis that they induce mucosal inflammation by directly increasing chemotaxis in vivo.
\end{abstract}

\section{Introduction}

The leucocyte population of the normal human colon mucosa consists of lymphocytes, plasma cells, macrophages and variable numbers of eosinophils and mast cells. In health, there is a notable absence of neutrophils. In acute colitis caused by invasive enteric bacteria, a modest increase in the numbers of resident leucocytes is seen, but the predominant response is margination of neutrophils in dilated capillaries of the lamina propria and their migration in large numbers to the interstitium, with ultimate invasion of the epithelium and the formation of crypt abscesses. ${ }^{1,2}$ The initial chemotactic response of the neutrophils and other leucocytes to invasive organisms might be expected to influence the degree of inflammatory infiltration and thus the severity of the colitis. The leucocyte chemotactic response to non-invasive bacteria, which constitute normal colonic flora but which also may breach the epithelial barrier with injury, might logically be expected to be different from the leucocyte responses to their invasive counterparts. This possibility was tested by measuring chemotaxis

Received 10 May 1994; revised version accepted 22 Sept. 1994.

* Present address: Department of Mathematics, Millersville University, Millersville, PA, USA. by blood neutrophils and monocytes in response to Escherichia coli strains representing organisms with different virulence characteristics, such as the presence or absence of a 140-MDa invasive plasmid and a positive or negative Sereny test. In several experiments, eosinophil chemotactic responses were also documented. Statistical analysis evaluated both the system and biological variability of the in-vitro chemotactic assays and the response of leucocytes to the various bacterial chemo-attractants.

\section{Materials and methods}

\section{Chemotactic assays}

Chemotaxis was assayed in 48-well plates (Neuro Probe, Cabin John, MD, USA) by the method of Falk et $a l^{3}$ Responding leucocytes suspended in Gey's Balanced Salt Solution (GBSS; Gibco, Grand Island, NY, USA) containing bovine serum albumin (BSA; Sigma, MO, USA) $1 \%$ were placed in the upper, and the bacteria-conditioned media in the lower portion of the well, separated by a polycarbonate filter (Neuro Probe) with $3-\mu \mathrm{m}$ pores for neutrophils, $5-\mu \mathrm{m}$ pores for monocytes and 8- $\mu \mathrm{m}$ pores for eosinophils. Plates were incubated at $37^{\circ} \mathrm{C}$ in $\mathrm{CO}_{2} 5 \%$ for $30 \mathrm{~min}$ 
(neutrophils) or $90 \mathrm{~min}$ (monocytes and eosinophils). Assays were conducted in triplicate with GBSS-BSA and Mueller-Hinton (M-H) bacterial culture medium (Scott Laboratories, Fiskeville, RI, USA) as negative controls and $10^{-5} \mathrm{M} \mathrm{N}$-formyl-met-phe (FMP; Sigma) as a standard chemo-attractant in each plate. After incubation, filters were drained, air dried and stained with Diff-Quik (AHS del Caribe Inc., Aguada, PR) for microscopic enumeration of migrating cells. Cells moving through the filter were counted in 30 microscopic fields under high power and averaged. Mean values for cells responding to the GBSS-BSA and $\mathrm{M}-\mathrm{H}$ broth were subtracted from the means responding to the test chemo-attractants and FMP. Results were expressed as the mean number of cells migrating, and also as the percentage of the response to FMP. Checkerboard experiments were performed with $100 \%, 20 \%, 10 \%$ and $5 \%$ concentrations of the five chemo-attractants (A, B, C, D, H; table I) above and below the filters to differentiate random migration of neutrophils from directed chemotaxis. ${ }^{4}$

\section{Isolation of responding leucocytes}

Neutrophils, monocytes and eosinophils were separated from $50 \mathrm{ml}$ of heparinised blood from normal volunteers. Erythrocytes were sedimented by the addition of dextran (Macrodex, Pharmacia) $6 \%$ and monocytes were separated from neutrophils by density gradient sedimentation on Ficoll-sodium diatrizoate (Histopaque-1077; Sigma) at $300 \mathrm{~g}$ for $30 \mathrm{~min}$ at $24^{\circ} \mathrm{C}$. Residual erythrocytes were cleared from the neutrophil pellet by the addition of ammonium chloride lysing buffer. ${ }^{5}$ Eosinophils from three subjects were isolated from neutrophils by gradient centrifugation on metrizamide (Sigma) $22 \%$ at $1200 \mathrm{~g}$ for $45 \mathrm{~min}^{6}{ }^{6}$ Cell preparations were washed, resuspended and counted in a haemacytometer. Cytocentrifuge preparations were stained with Diff-Quik and examined microscopically for cell purity.

\section{Bacterial chemo-attractants}

The eight bacteria listed in table I were used to generate conditioned medium for testing the chemotactic responses of the leucocyte populations. The first five organisms were isolated from patients with acute dysentery. E. coli strains 497-78, 978-77, 5898-71 and 3189-75 were obtained from I. Kaye Wachsmuth, Centers for Disease Control, Atlanta, GA, where the Sereny tests were done. Strains $4608-58$ and its isogenic plasmid-free mutant 4608-50A4 were obtained from T. L. Hale, Walter Reed Army Institute for Research, Washington, D.C. A. Wanger, University of Texas, Houston, TX, kindly performed the plasmid analysis of the other organisms. The E. coli strain $\mathrm{O} 6-\mathrm{H} 1$ was capsulate and contained a haemolysin. Strain ON-2 was obtained from P. K. Peterson, University of Minnesota, Minneapolis, MN. Conditioned media were produced by inoculating $10^{7}$. bacteria into separate culture flasks containing M-H broth and culturing for $16 \mathrm{~h}$ to stationary phase. The bacteria were then removed by centrifugation and the supernate was passed through a 200-nm filter; it was subdivided into convenient samples and frozen until use in the chemotactic assays. In a number of experiments $(n=4-8)$ chemo-attractant activity of each undiluted conditioned medium was compared to that of a 1 in 10 dilution.

\section{Statistical analysis}

To identify the various factors influencing the results of the chemotactic assays, chemotactic responses of neutrophils and monocytes to GBSS-BSA and FMP were tested by analysis of variance with a nested design $^{7}$ to examine inter-subject, intra-subject, plateto-plate and well-to-well variation.

The inter- and intra-subject variations reflected biological factors, and the plate-to-plate and well-towell variations were of a technical nature. Results from 29 subjects, each contributing from 1 to nineteen assays, were used for the analysis. For each assay, from 1 to 3 plates were employed and each test variable was run in three adjacent wells. The total sample size for neutrophil experiments was 398 and for monocytes 577.

In the above nested design, the error factor for testing hypotheses is determined by the factor which is nested. For example, intra-subject variation was the error factor for inter-subject differences; plate-to-plate variation was used to test intra-subject differences and well-to-well variation was the plate-to-plate error term. Thus, the method of analysis needs to be considered when interpreting the results.

A randomised block design ${ }^{7}$ was used to test for significant differences of neutrophil and monocyte responses to the eight $E$. coli-conditioned media. Cells from a single subject were tested in one plate with three wells for each chemo-attractant. Eight subjects were tested in all. Separate statistical analyses were conducted for the number of cells migrating and for the number expressed as a percentage of the response to FMP. Eosinophil chemotaxis results were not analysed statistically because cells from only three donors were assayed.

\section{Results}

The chemotactic responses of neutrophils and monocytes to each bacterial chemo-attractant were significantly greater $(p<0.05)$ than to GBSS-BSA and unconditioned M-H broth (table II) by Duncan's multiple range test. ${ }^{7}$ Neutrophil chemotaxis in response to conditioned media ranged from $65.9 \%$ to $157.8 \%$ of the response to FMP, and monocyte responses varied from $92.9 \%$ to $114.8 \%$ of those to the FMP standard (table III). As regards the invasive $E$. coli strains, medium conditioned by strain $5898-71$ 
Table I. Characteristics of $E$. coli used to produce media with chemo-attractant activity

\begin{tabular}{lllcc}
\hline & Serotype & Strain no. & $\begin{array}{c}\text { 140-MDa } \\
\text { plasmid }\end{array}$ & $\begin{array}{c}\text { Sereny } \\
\text { test }\end{array}$ \\
\hline A. 028 acNM & $497-78$ & + & + \\
B. 029 NM & $978-77$ & + & - \\
C. 0124 H30 & $5898-71$ & + & + \\
D. 0124 HNT & $3189-75$ & + & - \\
E. 0143 & $4608-58$ & + & NT \\
F. 0143 & $4608-58$ A4 & - & NT \\
G. 25922 ATCC & O6-H1 & - & NT \\
H. O22.H16 & ON-2 & $-*$ & NT \\
\hline
\end{tabular}

NT, not tested.

* 120-MDa plasmid.

Table II. Leucocyte migration in response to conditioned culture media, controls and FMP standard

\begin{tabular}{lccc}
\hline & & \multicolumn{2}{c}{ Mean (SD) number of migrating } \\
\cline { 3 - 4 } Medium & $\begin{array}{c}\text { Number of } \\
\text { subjects }\end{array}$ & neutrophils & monocytes \\
\hline $497-78$ & & $126 \cdot 1(61 \cdot 5)$ & $114 \cdot 8(77 \cdot 1)$ \\
$978-77$ & 8 & $152 \cdot 3(85 \cdot 4)$ & $126 \cdot 7(112 \cdot 6)$ \\
$5898-71$ & 8 & $205 \cdot 1(96 \cdot 7)$ & $145 \cdot 6(110 \cdot 4)$ \\
$3189-75$ & 8 & $142 \cdot 2(88 \cdot 9)$ & $142 \cdot 2(132 \cdot 8)$ \\
$4608-58$ & 8 & $197 \cdot 8(64 \cdot 3)$ & $191 \cdot 7(175 \cdot 9)$ \\
$4608-58 A 4$ & 4 & $167 \cdot 0(84 \cdot 2)$ & $202 \cdot 3(67 \cdot 1)$ \\
O6-H1 & 4 & $275 \cdot 8(132 \cdot 7)$ & $161 \cdot 1(150 \cdot 6)$ \\
ON-2 & 8 & $245 \cdot 2(117 \cdot 7)$ & $155 \cdot 7(154 \cdot 4)$ \\
GBSS-BSA & 8 & $22 \cdot 1(8 \cdot 4)$ & $21 \cdot 8(13 \cdot 2)$ \\
M-H broth & 12 & $69 \cdot 5(36 \cdot 3)$ & $66 \cdot 8(33 \cdot 4)$ \\
FMP & 12 & $212 \cdot 8(128 \cdot 1)$ & $153 \cdot 1(125 \cdot 8)$ \\
& 12 & & \\
\hline
\end{tabular}

Table III. Chemotaxis expressed as a percentage of cells migrating to FMP

\begin{tabular}{|c|c|c|c|}
\hline \multirow{2}{*}{ Medium } & \multirow{2}{*}{$\begin{array}{c}\text { Number of } \\
\text { subjects }\end{array}$} & \multicolumn{2}{|c|}{ Mean (SD) percentage of migrating } \\
\hline & & neutrophils & monocytes \\
\hline $497-78$ & 8 & $65.9(28.9)$ & $92 \cdot 9(37 \cdot 3)$ \\
\hline $978-77$ & 8 & $80 \cdot 5(46 \cdot 7)$ & $94 \cdot 8(41 \cdot 2)$ \\
\hline $5898-71$ & 8 & $103 \cdot 3(38 \cdot 1)$ & $107.8(52.0)$ \\
\hline $3189-75$ & 8 & $88 \cdot 0(36 \cdot 6)$ & $101 \cdot 5(52 \cdot 9)$ \\
\hline $4608-58$ & 4 & $157.8(89.6)$ & $94.6(10 \cdot 8)$ \\
\hline $4608-58 \mathrm{~A} 4$ & 4 & $145 \cdot 1(81 \cdot 1)$ & $109 \cdot 4(33 \cdot 2)$ \\
\hline O6-H1 & 8 & $141 \cdot 4(83 \cdot 3)$ & $114.8(56.9)$ \\
\hline ON-2 & 8 & $126 \cdot 1(48 \cdot 9)$ & $111 \cdot 3(46 \cdot 9)$ \\
\hline
\end{tabular}

was the most chemo-attractive for neutrophils, but some of the most marked neutrophil chemotactic activity occurred toward organisms not associated with enteric invasion and also to one of the invasive organisms stripped of its invasion plasmid. For example, the response of neutrophils to the non-invasive strains $\mathrm{ON} 2$ and $\mathrm{O} 6 \mathrm{H} 1$ was distinctly greater than to the enteroinvasive strains 497-78, 978-77 and 3189-71. Monocytes exhibited somewhat less variability in their chemotactic activity.

The mean numbers of monocytes and neutrophils attracted by undiluted conditioned media from the eight bacteria were invariably greater than attracted
Table IV. Chemotaxis of eosinophils in response to conditioned culture medium

\begin{tabular}{lcccc}
\hline Medium & $\begin{array}{c}\text { Number } \\
\text { of } \\
\text { subjects }\end{array}$ & $\begin{array}{c}\text { Mean (SD) } \\
\text { number of } \\
\text { cells migrating }\end{array}$ & $\begin{array}{c}\text { Number } \\
\text { of } \\
\text { subjects }\end{array}$ & $\begin{array}{c}\text { Mean (SD) } \\
\text { percentage of } \\
\text { FMP response }\end{array}$ \\
\hline $497-78$ & 3 & $38 \cdot 6(19 \cdot 8)$ & 2 & $63 \cdot 2(10 \cdot 0)$ \\
$978-77$ & 3 & $42 \cdot 3(27 \cdot 9)$ & 2 & $78 \cdot 0(21 \cdot 9)$ \\
$5898-71$ & 3 & $60 \cdot 7(21 \cdot 1)$ & 2 & $93 \cdot 8(26 \cdot 5)$ \\
$3189-75$ & 3 & $42 \cdot 4(24 \cdot 5)$ & 2 & $73 \cdot 0(17 \cdot 3)$ \\
O6-H1 & 3 & $46 \cdot 2(26 \cdot 0)$ & 2 & $80 \cdot 0(20 \cdot 1)$ \\
ON-2 & 3 & $35 \cdot 6(17 \cdot 4)$ & 2 & $58 \cdot 7(9 \cdot 1)$ \\
GBSS-BSA & 3 & $15 \cdot 0(5 \cdot 4)$ & $\ldots$ & $\ldots$ \\
FMP & 2 & $81 \cdot 7(19 \cdot 5)$ & $\ldots$ & $\ldots$ \\
M-H broth & 3 & $18 \cdot 8(7 \cdot 7)$ & $\ldots$ & $\ldots$ \\
\hline
\end{tabular}

by the 1 in 10 dilutions, but the differences were not statistically significant. Checkerboard experiments indicated directed chemotaxis in response to each conditioned medium tested, but high concentrations invoked some random movement. When all checkerboard experiments are considered, approximately onethird of cell migration was random, and two-thirds was directed. Eosinophil responses were less than those of neutrophils and monocytes (table IV). Except for a somewhat more active response to broth conditioned by virulent strain 5898-71, eosinophils did not discriminate between the media conditioned by the various bacteria.

For experiments evaluating system variations, the mean chemotactic responses of neutrophils to GBSSBSA was 25.3 cells $(n=133)$ and that of monocytes was 19.5 cells $(n=192)$. The mean number of neutrophils migrating in response to the $10^{-5} \mathrm{M}$ FMP standard was 255.3 , and of monocytes 168.7 cells. Thus the response to GBSS-BSA for neutrophils was $10.1 \%$ and for monocytes $11.6 \%$ of the respective responses to FMP. On the basis of these data, statistical analysis (tables V and VI) indicated that differences in intrasubject responses of neutrophils and monocytes accounted for most of the variability in the chemotaxis assay system. Therefore, we have not included replicate data from the same subject in the analysis of responses to bacterial chemo-attractants. The next most important factor was plate-to-plate variation, which led us to express results as a percentage of the response to the FMP standard of each plate in addition to indicating the absolute number of cells migrating. Variation due to the technical factor of well-to-well differences, and the biological effects of person-toperson variations were negligible relative to intrasubject variations.

\section{Discussion}

Certain methodological aspects of this study deserve mentioning. In preliminary work we tested the chemoattractant properties of bacterial lysates, and found them considerably less active than the culture supernates. We also found that M-H broth was somewhat 
Table V. Analysis of variance of the number of leucocytes migrating to buffer (GBSS)

\begin{tabular}{lrrrr}
\hline Source of variation & df & Variance & F & p value \\
\hline Neutrophils & & & & \\
Subject-to-subject & 26 & 3161.8 & 1.00 & 0.490 \\
Intra-subject & 39 & 3146.5 & 9.15 & $<0.001$ \\
Plate-to-plate & 64 & 343.9 & 11.99 & $<0.001$ \\
Well-to-well & 259 & 28.7 & $\ldots$ & $\ldots$ \\
Monocytes & & & & \\
Subject-to-subject & 28 & 304.8 & 0.49 & 0.979 \\
Intra-subject & 55 & 618.3 & 7.57 & $<0.001$ \\
Plate-to-plate & 103 & 81.7 & 5.07 & $<0.001$ \\
Well-to-well & 373 & 16.1 & $\ldots$ & $\ldots$ \\
& & & & $\ldots$
\end{tabular}

df, degrees of freedom; variance, mean square of the differences from the means; F, F statistic.

chemo-attractive, therefore all results with culture supernates have the $\mathrm{M}-\mathrm{H}$ activity subtracted. The statistical analysis indicated considerable variation in chemotaxis of leucocytes of the same person studied at different times relative to the variation between different people. Thus, the variation in chemotactic responses of leucocytes from the same person at different times must be taken into consideration in any analysis of such data. In addition, plate-to-plate variation in similar assays should be eliminated by expressing the results of each plate in terms of its standard.

These studies documented the in-vitro chemotactic responses of blood neutrophils, monocytes and eosinophils to media conditioned by various strains of $E$. coli with different virulence characteristics. The organisms tested included two enteroinvasive strains that produced kerato-conjunctivitis (Sereny positive) in guinea-pigs and two others that did not, and a virulent strain with a 140-MDa invasion plasmid and its noninvasive isogenic mutant stripped of its plasmid. The two other organisms tested were also not enteroinvasive. The greatest in-vitro chemotactic activity was in response to medium from bacteria lacking in an important invasion characteristic or to those not known to be enteroinvasive. Conversely, the neutrophils, monocytes and to some extent eosinophils

\section{References}

1. Jass JR. The large intestine. In: Morson BC (ed) Alimentary tract vol 3 of Symmers W St C (ed) Systemic pathology, 3rd edn. Edinburgh, Churchill Livingstone. 1987:313-395.

2. Whitehead R. Mucosal biopsy of the gastrointestinal tract. In: Bennington JL (ed) Major problems of pathology, vol 3. Philadelphia, W.B. Saunders Co. 1985: 209.

3. Falk W, Goodwin RH, Leonard EJ. A 48-well micro-chemotaxis assembly for rapid and accurate measurement of leukocyte migration. J Immunol Methods 1980; 33: 239247.

4. Zigmond SH, Hirsch JG. Leukocyte locomotion and chemotaxis: New methods for evaluation and demonstration of a cell-derived chemotactic factor. $J$ Exp Med 1973; 137: $387-410$.
Table VI. Analysis of variance of the number of leucocytes migrating to FMP

\begin{tabular}{lrrrr}
\hline Source of variation & df & Variance & F & p value \\
\hline Neutrophils & & & & \\
Subject-to-subject & 26 & $44061 \cdot 1$ & 1.03 & 0.456 \\
Intra-subject & 42 & $42672 \cdot 2$ & 6.82 & $<0.001$ \\
Plate-to-plate & 64 & $6252 \cdot 8$ & 15.30 & $<0.001$ \\
Well-to-well & 265 & $408 \cdot 8$ & $\ldots$ & $\ldots$ \\
Monocytes & & & & \\
Subject-so-subject & 28 & $13210 \cdot 8$ & 0.69 & 0.857 \\
Intra-subject & 57 & $19267 \cdot 3$ & $23 \cdot 29$ & $<0.001$ \\
Plate-to-plate & 107 & 828.2 & 9.33 & $<0.001$ \\
Well-to-well & 384 & 88.8 & $\ldots$ & $\ldots$ \\
\end{tabular}

df, degrees of freedom; variance, mean square of difference from the mean; F, F statistic.

exhibited less chemotaxis to the more virulent organisms. If these in-vitro results can be extrapolated to invivo responses, factors other than the virulence characteristics of bacteria govern the magnitude of the chemotactic response of neutrophils, monocytes and eosinophils in inflammation due to invasive $E$. coli. The role of these agents in the secretion of the various chemotactic cytokines ${ }^{8}$ would be of interest.

In summary, active chemotaxis was exhibited by blood neutrophils, monocytes and eosinophils toward each $E$. coli-conditioned medium. However, chemotaxis was usually less vigorous toward media conditioned by enteroinvasive $E$. coli strains with recognised virulence factors, than to media conditioned by non-enteroinvasive strains. It is possible that an invading organism with diminished chemo-attractant properties might encounter a diminished or delayed leucocyte response, and thus have an advantage over more chemo-attractive bacteria. These experiments do not support the hypothesis that virulence factors of enteroinvasive $E$. coli contribute to mucosal inflammation by directly augmenting chemotactic responses by neutrophils, monocytes and eosinophils.

These studies were supported by PHS grant AM/AI 38223 and Biomedical Research Support Grant S-0705429. Ms C. Larson provided expert technical assistance.
5. Roos D, Loos JA. Changes in the carbohydrate metabolism of mitogenically stimulated human peripheral lymphocytes. 1. Stimulation by phytohaemagglutinin. Biochim Biophys Acta 1970; 222: 565-582.

6. Beeken WL, Northwood I, Beliveau C, Baigent G, Gump D. Eosinophils of human colonic mucosa: $\mathrm{C} 3 \mathrm{~b}$ and Fc gamma receptor expression and phagocytic capabilities. Clin Immunol Immunopathol 1987; 43: 289-300.

7. Montgomery DC (ed). Design and analysis of experiments, $3 \mathrm{rd}$ edn. New Yark, John Wiley and Sons. 1991: 75, 134-169, 439-456.

8. Miller MD, Krangel MS. Biology and biochemistry of the chemokines: a family of chemostatic and inflammatory cytokines. Crit Rev Immunol 1992; 12: 17-46. 